\title{
A Review of Evaluation Techniques for Social Dialogue Systems
}

\author{
Extended Abstract \\ Helen Hastie \\ Interaction Lab \\ Heriot-Watt University \\ Edinburgh, U.K. \\ h.hastie@hw.ac.uk
}

Amanda Cercas Curry

Interaction Lab

Heriot-Watt University

Edinburgh, U.K.

ac293@hw.ac.uk

\author{
Verena Rieser \\ Interaction Lab \\ Heriot-Watt University \\ Edinburgh, U.K. \\ v.t.rieser@hw.ac.uk
}

\begin{abstract}
In contrast with goal-oriented dialogue, social dialogue has no clear measure of task success. Consequently, evaluation of these systems is notoriously hard. In this paper, we review current evaluation methods, focusing on automatic metrics. We conclude that turnbased metrics often ignore the context and do not account for the fact that several replies are valid, while end-of-dialogue rewards are mainly hand-crafted. Both lack grounding in human perceptions.
\end{abstract}

\section{CCS Concepts}

- Human-centered computing $\rightarrow$ Natural language interfaces; - General and reference $\rightarrow$ Surveys and overviews; Evaluation;

\section{Keywords}

Automatic Evaluation, Social Dialogue Systems, Conversational Agents, Evaluation Metrics

\section{ACM Reference Format:}

Amanda Cercas Curry, Helen Hastie, and Verena Rieser. 2017. A Review of Evaluation Techniques for Social Dialogue Systems: Extended Abstract. In Proceedings of 1st ACM SIGCHI International Workshop on Investigating Social Interactions with Artificial Agents (ISIAA'17). ACM, New York, NY, USA, 2 pages. https://doi.org/10.1145/3139491.3139504

\section{Introduction}

Non-task-oriented, social dialogue systems, aka "chatbots", receive an increasing amount of attention as they are designed to establish a rapport with the user or customer, providing engaging and coherent dialogue. Traditional dialogue systems $[8,10]$ tend to be task-orientated for a limited domain and evaluation methods of such systems have been much researched (see [1] for an overview). Evaluation of social dialogue systems, on the other hand, is challenging as there is no clear measure for task success and evaluating whether such a rapport has been established is far from clear-cut. One common method for evaluating such systems is human evaluation where subjects are recruited to interact with and rate different systems. However, human evaluation is highly subjective, time-consuming, expensive and requires careful design of the experimental set-up.

Permission to make digital or hard copies of part or all of this work for personal or classroom use is granted without fee provided that copies are not made or distributed for profit or commercial advantage and that copies bear this notice and the full citation on the first page. Copyrights for third-party components of this work must be honored.

For all other uses, contact the owner/author(s).

ISIAA'17, November 13, 2017, Glasgow, UK

(c) 2017 Copyright held by the owner/author(s)

ACM ISBN 978-1-4503-5558-2/17/11 . \$15.00

https://doi.org/10.1145/3139491.3139504
Table 1: Valid system response with low word overlap to reference.

\begin{tabular}{l}
\hline User utterance: \\
Have you read Murakami's new novel? \\
\hline Reference response: \\
No I don't think I have read Murakami's new \\
novel, what is it about? \\
\hline System output: \\
Yes, it wasn't my favourite but I still liked it. \\
\hline
\end{tabular}

\section{Automatic Metrics}

Automatic evaluation is popular because it is cost-effective and faster to run than human evaluation, and is needed for automatic benchmarking and tuning of algorithms. Here, we discuss existing automatic methods for developing social systems in terms of word-overlap metrics, machine learning-based estimation models and reward-based metrics. Since social systems lack a final success measure, many of the discussed metrics operate at turn-level.

\subsection{Word-Overlap Metrics}

Word-overlap metrics, such as BLEU [9] and ROUGE [4], are borrowed from Machine Translation (MT) and Summarisation and have been widely been used to evaluate neural dialogue system output, as reported in, for example [3,11]. However, these metrics have not been shown to correlate well with human judgements in a dialogue setting [5]. One possible explanation is that there is no "gold standard" to compare with, as in MT: there may be many valid responses to an utterance that have no or few overlapping $\mathrm{n}$-grams and would thus receive low BLEU or ROUGE scores, see example in Table 1. Measures from information theory such as perplexity have also been used for evaluation, e.g. comparing neural models to n-grams [12], however perplexity can be difficult to interpret. There is, therefore, a need for an evaluation method that does not measure success by comparing an utterance to humangenerated responses but instead considers the utterance itself and its appropriateness within its context.

\subsection{Machine Learning Methods for Dialogue Evaluation}

Recently, Machine Learning (ML) based evaluation has gained popularity. This method operates on the turn-level and aims to provide an estimation model of a "good" response. The advantages of this method is that it has been shown to come closer to humangenerated responses [6] than BLEU and ROUGE. However, such methods require retraining for each domain. 
Discriminative Models: These models attempt to distinguish the "right" from the "wrong" answer. Next-Utterance Classification (NUC) [7] can be evaluated by measuring the system's ability to select the next answer from a list of possible answers sampled from elsewhere in the corpus, using retrieval metrics such as recall. NUC offers several advantages: performance is easy to compute automatically and the task is interpretable and can be easily compared to human performance. However, similar issues to wordbased metrics do apply in that there is not necessarily one single correct answer.

More recently, adversarial evaluation measures have been proposed to distinguish a dialogue model's output from that of a human. For example, the model proposed by [2] achieves a $62.5 \%$ success rate using a Recurrent Neural Networks (RNN) trained on email replies.

Classification Models: [6] propose to predict human scores from a large dataset of human ratings of Twitter responses. The proposed models learn distributed representations of the context, reference response and the system's response using a hierarchical RNN encoder. The learned model correlates with human scores at the turn level and also generalises to unseen data. However, it does tend to have a bias towards generic responses.

\subsection{Reward-based Metrics}

Reinforcement Learning (RL) based models have been applied to task-based systems [10] to optimise interaction for some reward. For social systems, this has also been investigated as a means to avoid generic responses, such as "I don't know". Here, the evaluation function is implemented as the reward. We will discuss these types of reward at turn-level and at system-level.

Turn-level rewards: [3] propose a metric involving a weighted sum of three measures:

- Coherence: semantic similarity between consecutive turns,

- Information flow: semantic dissimilarity between utterances of the same speaker,

- Ease of answering: negative log-likelihood of responding to an utterance with a dull response (as defined by a blacklist).

In their experiments, they find the RL approach outperforms their other systems in terms of dialogue length, diversity of answers and overall quality of multi-turn dialogues. This suggests that the proposed reward function successfully captures the relationship between an utterance and a response at least partially, which can be useful in evaluating potential responses without the need for human-generated references. However, while coherence at the turnlevel is a key factor in quality estimation, it does not necessarily reflect the overall quality of the dialogue.

System-level rewards: The reward function by [3] was based on heuristics, whereas [13] use a Wizard-of-Oz experiment to measure engagement and deduct a reward function with the following metrics:

- Conversational depth: the number of consecutive turns belonging to the same topic,
- Lexical diversity/information gain: the number of unique words that are introduced into the conversation from both the system and the user,

- Overall dialogue length.

\section{Conclusion and Discussion}

It is clear that there is still work to be done with respect to establishing an effective evaluation method that can capture all aspects of dialogue from naturalness and coherence to long-term engagement and flow. Word-based metrics such as BLEU, ignore the fact there may be any number of equally valid and appropriate responses, while turn-based metrics do not account for the overuse of generic responses, and system-level rewards are based on heuristics. In future work, we will utilise data we gathered as part of the Amazon Alexa Prize challenge to build a data-driven model to predict customer ratings.

\section{Acknowledgements}

This research is supported by Rieser's EPSRC projects DILiGENt (EP/M005429/1) and MaDrIgAL (EP/N017536/1); and from the RAEng/Leverhulme Trust Senior Research Fellowship Scheme (Hastie/LTSRF1617/13/37).

\section{References}

[1] Helen Hastie. 2012. Metrics and evaluation of spoken dialogue systems. In Data-Driven Methods for Adaptive Spoken Dialogue Systems. Springer New York, 131-150.

[2] Anjuli Kannan and Oriol Vinyals. 2017. Adversarial Evaluation of Dialogue Models. CoRR abs/1701.08198 (2017). http://arxiv.org/abs/1701.08198

[3] Jiwei Li, Will Monroe, Alan Ritter, Michel Galley, Jianfeng Gao, and Dan Jurafsky. 2016. Deep Reinforcement Learning for Dialogue Generation. In Proceedings of Empirical Methods in Natural Language Processing (EMNLP).

[4] Chin-Yew Lin. 2004. ROUGE: A package for automatic evaluation of summaries. In Text summarization branches out: Proceedings of the ACL-04 workshop. Barcelona, Spain, 74-81. http://aclweb.org/anthology/W04-1013

[5] Chia-Wei Liu, Ryan Lowe, Iulian Serban, Michael Noseworthy, Laurent Charlin, and Joelle Pineau. 2016. How NOT To Evaluate Your Dialogue System: An Empirical Study of Unsupervised Evaluation Metrics for Dialogue Response Generation. In Proceedings of Empirical Methods in Natural Language Processing $(E M N L P)$. Austin, TX, USA. arXiv:1603.08023.

[6] Ryan Lowe, Michael Noseworthy, Iulian Serban, Nicolas Angelard-Gontier, Yoshua Bengio, and Joelle Pineau. 2017. Towards an Automatic Turing Test: Learning to Evaluate Dialogue Responses. In (under review).

[7] Ryan Lowe, Iulian Vlad Serban, Michael Noseworthy, Laurent Charlin, and Joelle Pineau. 2016. On the Evaluation of Dialogue Systems with Next Utterance Classification. In Proceedings of the SIGDIAL 2016 Conference, The 17th Annual Meeting of the Special Interest Group on Discourse and Dialogue, 13-15 September 2016, Los Angeles, CA, USA. 264-269. http://aclweb.org/anthology/W/W16/W16-3634.pdf

[8] Mike McTear. 2004. Spoken Dialogue Technology: Toward the Conversational User Interface. Springer, London.

[9] Kishore Papineni, Salim Roukos, Todd Ward, and Wei-Jing Zhu. 2002. BLEU: A Method for Automatic Evaluation of Machine Translation. In Proceedings of Annual Meeting of the Association for Computational Linguistics (ACL) (ACL '02). 311-318.

[10] Verena Rieser and Oliver Lemon. 2011. Reinforcement Learning for Adaptive Dialogue Systems: A Data-driven Methodology for Dialogue Management and Natural Language Generation. Book Series: Theory and Applications of Natural Language Processing, Springer, Berlin/Heidelberg.

[11] Alessandro Sordoni, Michel Galley, Michael Auli, Chris Brockett, Yangfeng Ji, Margaret Mitchell, Jian-Yun Nie, Jianfeng Gao, and Bill Dolan. 2015. A Neural Network Approach to Context-Sensitive Generation of Conversational Responses. In Proceedings of the 2015 Conference of the North American Chapter of the Association for Computational Linguistics: Human Language Technologies (NAACL-HLT). Denver, Colorado.

[12] Oriol Vinyals and Quoc Le. 2015. A neural conversational model. arXiv preprint arXiv:1506.05869 (2015).

[13] Zhou Yu, Ziyu Xu, Alan W. Black, and Alexander I. Rudnicky. 2016. Strategy and Policy Learning for Non-Task-Oriented Conversational Systems. In Proceedings of the SIGDIAL. 